\title{
Zur neurovaskulären Problematik von Krawatten
}

\author{
M. Spitzer, Ulm
}

Mein Freund Eckart von Hirschhausen wusste es schon lange, der Chef von Mercedes, Dieter Zetsche auch; die Krawattenabteilung von Breuninger, Stuttgart, hat es daher schon vor der empirischen Bestätigung am härtesten getroffen: Krawatten reduzieren die Gehirndurchblutung.

Im Bereich der Medizin wird das Tragen von Krawatten schon länger problematisiert, vor allem jedoch aus infektiologischer Sicht: Schon vor mehr als 10 Jahren hatte man auf etwa 50\% der Krawatten von Ärzten Krankheitserreger gefunden, u.a. auch Staphylococcus aureus, Klebsiella pneumoniae und Pseudomonas aeruginosa und andere (2). Nach einer neuen Metaanalyse der relevanten Studien hierzu (es sind nur insgesamt sechs) ist der Evidenzgrad für eine klinische Relevanz dieser Tatsache jedoch als eher gering einzustufen (4).

Bereits im Jahr 2003 wurde zudem publiziert, dass Krawatten den Augeninndruck steigern können und damit möglicherweise das Risiko für die Entstehung eines Glaukoms („grüner Star"). Messungen des Augeninnendrucks bei 20 gesunden Probanden und 20 Patienten mit Glaukom ergaben eine Steigerung des Augeninnendrucks durch eine eng getragene Krawatte um 2,6 mmHg bzw. 1,0 $\mathrm{mmHg}$ (6).

Die neurovaskuläre Seite des Tragens von Krawatten wurde jedoch erst kürzlich mit den Methoden der modernen Neurowissenschaft näher beleuchtet. Robin Lüddecke von der Universität Schleswig-Holstein in Kiel bestimmte den arteriellen und venösen intrakraniellen Blutfluss bei insgesamt 30 jungen gesunden männlichen Probanden im mittleren Alter von 24,6 $( \pm 2,9)$ Jahren. Sie wurden randomisiert in zwei

Nervenheilkunde 2018; 37: 655-656

Korrespondenzadresse

Prof. Dr. Dr. Manfred Spitzer, Universitätsklinikum UIm Klinik für Psychiatrie und Psychotherapie III

Leimgrubenweg 12, $89075 \mathrm{Ulm}$
Gruppen aufgeteilt, wobei die eine Gruppe dreimal ohne Krawatte und die andere Gruppe zunächst einmal mit lockerer Krawatte, dann mit „leicht unangenehm enger" Krawatte und dann noch einmal 15 Minuten später (bei wieder geöffneter Krawatte) eine Magnetresonanztomographie (MRT) des Gehirns erhielt. Die Messungen dauerten jeweils 15 Minuten. Mittels arterial spin labeling (ASL) wurde dabei der zerebrale Blutfluss (cerebral blood flow, $\mathrm{CBF}$ ) quantifiziert. Mittels quantitativer Phasenkontrast-Angiographie (PCA) wurde der venöse Blutfluss der Jugularvenen über einen kompletten Pulsschlag (RRcycle) gemessen. Zudem wurden strukturelle Aufnahmen gemacht, was eine Segmentierung des Gehirns in graue und weiße Substanz ermöglichte. Daraus wurde eine Maskierung der funktionellen mittels ASL durchgeführten Gehirnschnittbilder möglich, sodass die Berechnung des arteriellen Blutflusses in der gesamten grauen Substanz (whole-brain gray matter perfusion) möglich wurde.

Hierbei zeigte sich eine signifikante $\mathrm{Ab}$ nahme des zerebralen Blutflusses (im Vergleich zu Baseline) nach Zuziehen der Krawatte um 7,5\% $(-4,33 \pm 3,34 \mathrm{ml} / \mathrm{min}$ pro 100 g graue Substanz; $\mathrm{p}<0,001)$ und da- nach (wieder geöffnete Krawatte) eine weitere Abnahme um 5,7\% $(-3,08 \pm 1,52 \mathrm{ml} /$ min pro $100 \mathrm{~g}$ graue Substanz; $\mathrm{p}<0,001)$. Dieser Abfall wurde bei 13 von 15 (86,6\%) der Gruppe mit straff gebundener Krawatte beobachtet, während in der Kontrollgruppe bei 5 Probanden (33,3\%) der Blutfluss abfiel und bei 6 Probanden (40\%) anstieg. Die übrigen 6 Probanden (aus der Gesamtgruppe) zeigten praktisch keine Veränderungen (weniger als $1 \mathrm{ml} / \mathrm{min} / 100$ g) des zerebralen Blutflusses ( $\downarrow$ Abb. 1).

In der quantitativen Angiographie zeigte sich keine Veränderung des venösen Blutflusses.

Die Autoren diskutieren ihre Ergebnisse dahingehend, dass die Verminderung des CBF noch im physiologischen Rahmen liege und nur bei 6 Probanden größer als 10\% war. Aus klinischer Sicht könnten Verminderungen des CBF nur bei älteren Menschen, starken Rauchern oder Menschen mit vaskulären Vorschädigungen jedoch relevant sein. Sie schließen ihre Diskussion mit den Worten: „Im Lichte dieser Befunde sind wir froh darüber, dass Palazzo und Hocken bereits geklärt haben, dass Patienten von Ärzten nicht [das Tragen von Kra-
Abb. 1

Mittlerer zerebraler Blutfluss (CBF) in Prozent des Ausgangswerts (Baseline) mit enger Krawatte und 15 Minuten nach Öffnen der Krawatte (nach Daten aus 3, Tab. 1).

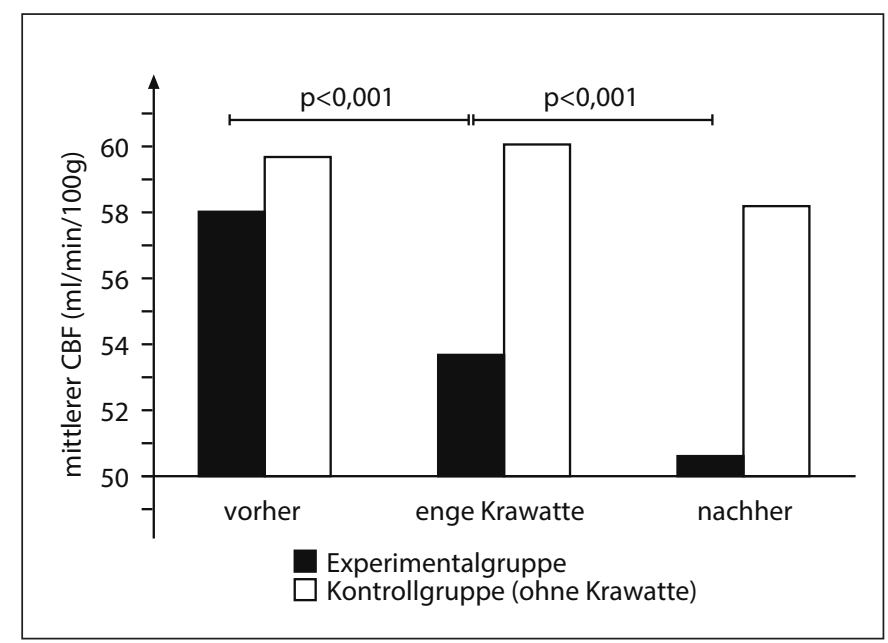


watten] als Dresscode erwarten“ (3, S. 864, Übersetzung durch den Autor). Die genannten Autoren hatten dies in einer Studie zu den Erwartungen bezüglich der adäquaten Kleidung von Ärzten bei 75 Patienten des Great Western Hospital im britischen Swindon erfragt. ,... neckties ... were not expected“ (5, S. 30).

Warum sollte $\operatorname{man}(\mathrm{n})$ überhaupt eine Krawatte tragen? Dazu kann man in einem Leserbrief von Brian Horton aus Tasmanien, der am 28. Juli 2018 im Fachblatt New Scientist publiziert wurde, das Folgende nachlesen: „Der beste Grund, der mir für das Tragen einer Krawatte je zu Ohren kam, besteht darin, dass sie einen von ei- nem normalen Arbeiter unterscheidet, der keine Krawatte trägt, weil sie von Maschinen erfasst werden könnte." Das macht durchaus Sinn, denn beim Bohren, Fräsen, am Fließband oder auf der Baustelle wäre es für den Träger einer Krawatte gefährlich, wenn die Krawatte erfasst wird und den Träger stranguliert oder in die Tiefe reißt. Durch das Tragen einer Krawatte deutet man also an, dass man „höhere“ Tätigkeiten ausführt. Oder, wie der Tasmanier sich direkt an Krawattenträger wendend, weiter auf den Punkt bringt: „Es ist ein Versuch, zu zeigen, dass Sie wichtig sind, der aber nur dann notwendig ist, wenn Sie keinerlei andere nützliche Fähigkeiten haben."

\section{Literatur}

1. Anonymus. Wearing a tie may hamper your brain. New Scientist 2018; 3186: 20.

2. Bhattacharya S. Doctors' ties harbour disease-causing germs. New Scientist 24.5.2004 https://www.news cientist.com/article/dn5029-doctors-ties-harbourdisease-causing-germs/; abgerufen am 4.8.2018.

3. Lüddecke $\mathrm{R}$, Lindner $\mathrm{T}$, Forstenpointner $\mathrm{J}$ et al Should you stop wearing neckties? - Wearing a tight necktie reduces cerebral blood flow. Neuroradiology 2018; 60: 861-864.

4. Pace-Asciak P, Bhimrao SK, Kozak FK, Westerberg BD. Health care professionals' neckties as a source of transmission of bacteria to patients: a systematic review. CMAJ Open 2018; 6: E26-E30.

5. Palazzo S, Hocken DB. Patients perspectives on how doctors dress. J Hosp Infect 2010; 74: 30-34.

6. Teng C, Gurses-Ozden R, Liebmann JM et al. Effect of a tight necktie on intraocular pressure. Br J Ophthalmol 2003; 87: 946-948. 